\title{
Three topics in online list coloring*
}

\author{
James Carraher, Sarah Loeb, Thomas Mahoney, \\ Gregory J. Puleo, Mu-Tsun Tsai, and Douglas B. West ${ }^{\dagger}$
}

\begin{abstract}
In online list coloring (introduced by Zhu and by Schauz in 2009), on each round the set of vertices having a particular color in their lists is revealed, and the coloring algorithm chooses an independent subset to receive that color. The paint number of a graph $G$ is the least $k$ such that there is an algorithm to produce a successful coloring with no vertex being shown more than $k$ times; it is at least the choice number. We study paintability of joins with complete or empty graphs, obtaining a partial result toward the paint analogue of Ohba's Conjecture. We also determine upper and lower bounds on the paint number of complete bipartite graphs and characterize 3-paint-critical graphs.
\end{abstract}

Keywords AND PHRASEs: Paintability, choosability, online list coloring, Ohba's Conjecture, complete bipartite graph.

\section{Introduction}

The list version of graph coloring, introduced by Vizing [18] and ErdősRubin-Taylor [2], has now been studied in hundreds of papers. Instead of having the same colors available at each vertex, each vertex $v$ has a set $L(v)$ (called its list) of available colors. An $L$-coloring is a proper coloring $f$ such that $f(v) \in L(v)$ for each vertex $v$. A graph $G$ is $k$-choosable if an $L$-coloring exists whenever $|L(v)| \geq k$ for all $v \in V(G)$. The choosability or choice number $\chi_{\ell}(G)$ is the least $k$ such that $G$ is $k$-choosable. Since the lists at vertices could be identical, always $\chi(G) \leq \chi_{\ell}(G)$. More generally, $G$ is $f$-choosable if a proper coloring can be chosen from the lists whenever $|L(v)|=f(v)$ for each vertex $v$.

An online version of list coloring was introduced by Zhu [20]; independently, Schauz [16] introduced an equivalent notion in a game setting. The lists are revealed one color at a time, by marking the vertices with that color

${ }^{*}$ Research of all authors partially supported by NSF grant DMS 08-38434 "EMSW21-MCTP: Research Experience for Graduate Students."

${ }^{\dagger}$ Research supported by NSA grant H98230-10-1-0363. 
in their lists. The coloring algorithm, which we call Painter, must choose an independent set of marked vertices to receive that color. Colored vertices will not be marked again; in essence, they are removed from the graph. The online choice number $\chi_{p}(G)$ (also called the paintability or paint number) is the least $k$ such that Painter can guarantee producing a proper coloring with no vertex marked more than $k$ times.

More generally, each vertex $v$ can tolerate being marked $f(v)$ times before it must be colored, and the graph is $f$-paintable if Painter can guarantee coloring the graph subject to these tolerances. When $G$ is $f$-paintable with $f(v)=k$ for all $v$, we say that $G$ is $k$-paintable; the online choice number is the least $k$ such that $G$ is $k$-paintable.

In the game setting, Painter has an adversary called Lister who specifies the marked sets. Again each vertex $v$ has a tolerance $f(v)$. Lister wins by marking some vertex $v$ more than $f(v)$ times; Painter wins by producing a proper coloring before this happens. To facilitate discussion, we imagine a pile of $f(v)$ tokens at $v$. When Lister marks $v$, one of these tokens is used, and Lister wins if $v$ is marked when it has no tokens.

The behavior of Lister models the need for Painter to win against "worstcase" presentation of lists in online list coloring, so the problems are equivalent. (Schauz originally called the players "Mr. Paint and Mrs. Correct"; "Marker/Remover" has also been used.)

If $G$ is not $f$-choosable, then $G$ is not $f$-paintable, since Lister can mimic a bad list assignment $L$ by marking in round $i$ the set $\{v \in V(G): i \in L(v)\}$; winning moves by Painter would form an $L$-coloring, which does not exist. In particular, $\chi_{p}(G) \geq \chi_{\ell}(G) \geq \chi(G)$. These values may all be distinct; they equal 4,3,2 for $K_{4, r}$ when $12 \leq r \leq 18$, for $K_{5, s}$ when $9 \leq s \leq 12$, and for $K_{6, t}$ when $8 \leq s \leq 10$.

Question 1.1. Does there exist $G$ such that $\chi_{p}(G)-\chi_{\ell}(G)$ is arbitrarily large? At least 2 ?

For example, let $G=K_{3, \ldots, 3}$ with $k$ parts. Kierstead [6] proved $\chi_{\ell}(G)=$ $\lceil(4 k-1) / 3\rceil$. Another proof appears in [9], where the authors also showed $\chi_{p}(G) \leq 3 k / 2$. However, it remains unknown whether $\chi_{p}(G)>\lceil(4 k-1) / 3\rceil$. Another candidate is $K_{m, m}$. Using the bound by Radhakrishnan and Srinivasan [15] on the number of edges needed to form a non- $k$-choosable $k$ uniform hypergraph, Alon observed that $\chi_{\ell}\left(K_{m, m}\right) \leq \lg m-\left(\frac{1}{2}-o(1)\right) \lg \lg m$, but [7] showed only $\chi_{p}\left(K_{m, m}\right) \leq \lg m$. As yet no graph $G$ is known with $\chi_{p}(G)-\chi_{\ell}(G)>1$.

Since $\chi_{\ell}(G) \geq \chi(G)$, a theme in the study of choosability has been to show that some earlier upper bound on $\chi(G)$ holds also for $\chi_{\ell}(G)$, thereby 
strengthening the earlier result. Since always $\chi_{p}(G) \geq \chi_{\ell}(G)$, paintability can be studied in a similar way. Schauz strengthened several choosability results to paintability, showing [16] that planar graphs are 5-paintable and that bipartite graphs are $\Delta(G)$-edge-paintable. In [17] and [3], he strengthened the Alon-Tarsi Theorem [1] to the setting of paintability by a purely combinatorial proof, and from this he obtained strengthenings to paintability of various choosability consequences of the Alon-Tarsi Theorem. In the language of online choosability, Zhu [20] characterized 2-paintable graphs and proved results about 3-paintability for complete bipartite graphs.

Since $\chi_{\ell}(G) \geq \chi(G)$, it is natural to ask when $\chi_{\ell}(G)=\chi(G)$; such graphs are chromatic-choosable. More restrictively, $G$ is chromatic-paintable if $\chi_{p}(G)=\chi(G)$. Ohba [11] conjectured that $G$ is chromatic-choosable when $|V(G)| \leq 2 \chi(G)+1$; after partial results in $[8,11,12,14]$, this was proved by Noel, Reed, and $\mathrm{Wu}$ [10]. Various researchers (see [7]) noted that the complete multipartite graph $K_{2, \ldots, 2,3}$ is chromatic-choosable but not chromaticpaintable, so the paintability analogue is different:

Conjecture 1.2. [5] If $|V(G)| \leq 2 \chi(G)$, then $G$ is chromatic-paintable.

In [5], the Combinatorial Nullstellensatz was used to show that $K_{2, \ldots, 2}$ and some other graphs with $|V(G)|=2 \chi(G)$ are chromatic-paintable. For $K_{2, \ldots, 2}$ this was reproved in [7] by an explicit strategy for Painter. We do not even know whether $|V(G)| \leq c \chi(G)$ implies $\chi_{p}(G)=\chi(G)$ for any $c \in(1,2]$.

Lacking such a result, we study lower-order terms. Ohba [11] proved that $G$ is chromatic-choosable when $|V(G)| \leq \chi(G)+\sqrt{2 \chi(G)}$. We strengthen and extend this in Section 2, proving that $G$ is chromatic-paintable when $|V(G)| \leq \chi(G)+2 \sqrt{\chi(G)-1}$. The join of graphs $G$ and $H$, denoted ${ }^{1} G \oplus H$, is obtained by adding to the disjoint union of $G$ and $H$ the edges $\{u v: u \in$ $V(G), v \in V(H)\}$. We show that if $G$ is $k$-paintable and $|V(G)| \leq \frac{t}{t-1} k$, then $\chi_{p}\left(G \oplus \bar{K}_{t}\right) \leq k+1$. The application then follows by induction on $\chi(G)$. We also prove for all $G$ that $G \uplus K_{t}$ is chromatic-paintable when $t$ is sufficiently large; this was proved independently by Kozik, Micek, and Zhu [9], who used it to obtain a slightly weaker strengthening of Ohba's result.

In Section 3, the general problem of $f$-paintability leads to a recurrence that provides an upper bound on the smallest $r$ such that $K_{k+j, r}$ is not $k$ paintable. This echoes both the elementary result by Vizing [18] that $K_{k, r}$ is $k$-choosable if and only if $r<k^{k}$ and the subsequent result by Hoffman

${ }^{1}$ This notation is new; it is consistent with the "Czech notation" introduced by Nešetřil in which the notation displays the result of the operation on $K_{2}$ and $K_{2}$, it evokes the additivity of the vertex sets, and it avoids conflicting with the proper use of "+" for disjoint union. 
and Johnson [4] that $K_{k+1, r}$ is $k$-choosable if and only if $r<k^{k}-(k-1)^{k}$. It turns out that $K_{k, r}$ is $k$-paintable if and only if $r<k^{k}$, but $K_{k+1, r}$ fails to be $k$-paintable when $r$ is smaller than $k^{k}-(k-1)^{k}$ by a constant fraction.

A graph is paint-critical if every proper subgraph has smaller paint number. Building on results in [19], in Section 4 we characterize paint-critical graphs with paint number 3 . The list is similar to the characterization of 3-choice-critical graphs by Voigt [19]. The 3-paint-critical graphs are the odd cycles, the complete bipartite graph $K_{4,2}$, "bicycles" (graphs consisting of two edge-disjoint cycles connected by one path of length at least 0 ), and certain "theta-graphs". The generalized theta-graph $\Theta_{\ell_{1}, \ldots, \ell_{k}}$ consists of two vertices joined by internally disjoint paths of lengths $\ell_{1}, \ldots, \ell_{k}$. The rest of the list of paint-critical graphs with paint number 3 consists of $\Theta_{2 r, 2 s, 2 t}$ and $\Theta_{2 r-1,2 s-1,2 t-1}$ with $r \geq 1$ and $s, t \geq 2$. Riasat and Schauz [13] independently explored 3-paint-critical graphs, giving for example a characterization of the minimal ones with respect to vertex deletion.

\section{Paintability of graph joins}

In this section we strengthen results of Ohba [11]. When describing strategies, we say that Lister marks or plays $M$ and that Painter deletes or colors an independent subset of $M$. When $f$ is a token assignment on $G$ and $H \subseteq G$, we say that $H$ is $f$-paintable when Painter has a winning strategy on $H$ for the restriction of $f$ to $V(H)$; that is, when each vertex of $H$ starts with $f(v)$ tokens. Let $d_{G}(v)$ denote the degree of vertex $v$ in a graph $G$.

Proposition 2.1. The following statements hold for Lister/Painter games.

(a) If $G$ is $f$-paintable, then every subgraph $H$ of $G$ is $f$-paintable.

(b) If $f(v)>d_{G}(v)$, then $G$ is $f$-paintable if and only if $G-v$ is $f$ paintable.

(c) If $G$ is not $f$-paintable, then Lister can force a win with no marked set before the last being independent.

Proof. (a) Edge deletion does not invalidate Painter moves, so we may let $H$ be an induced subgraph. In the game on $H$, Painter can respond to Lister's moves as in $G$ and win.

(b) By (a), we may assume that $G-v$ is $f$-paintable. To win on $G$, Painter follows a winning strategy for $G-v$, deleting $v$ when marked only if none of its neighbors are deleted by that strategy. In this way at most $d_{G}(v)$ tokens will be used at $v$, so Lister cannot win.

(c) If Lister plays an independent set $M$, then Painter can delete it, and Lister must then win on the remaining graph. Hence Lister can win by not playing $M$ and instead playing the rest of the winning strategy. 
The importance of Proposition 2.1(b) is that if in the course of the game on a graph $H$ a position arises in which a vertex has more remaining tokens than its remaining degree, then this vertex can be deleted without affecting who wins. For example, $d$-degenerate graphs (where every subgraph has a vertex of degree at most $d)$ are $(d+1)$-paintable. We thus invoke Proposition 2.1(b) to discard vertices by saying "by degeneracy".

The observations in Proposition 2.1 lead to our tool for extending Ohba's Theorem. In the special case $t=1$, the second condition is vacuous, implying (when iterated) that $G \diamond K_{r}$ is $(k+r)$-paintable when $G$ is $k$-paintable.

Theorem 2.2. If $G$ is $k$-paintable and $|V(G)| \leq \frac{t}{t-1} k$, then $G \oplus \bar{K}_{t}$ is $(k+1)$ paintable.

Proof. We give an explicit strategy for Painter. Let $T$ denote the added independent set of $t$ vertices. By Proposition 2.1(c), we may assume that Lister marks at least one vertex in $V(G)$ until no such vertices remain.

Let $\mathcal{S}$ be a winning strategy for Painter on $G$ with $k$ tokens at each vertex. Painter uses $\mathcal{S}$ until $V(G)$ is exhausted, except for one special round associated with the extra token at the vertices of $G$. When Lister plays $M$, say that the vertices of $T-M$ are omitted. Painter responds within $M \cap V(G)$ as specified by $\mathcal{S}$, unless condition $(*)$ holds:

Each vertex of $T-M$ was omitted at least $\mu$ times, where $\mu=\frac{k}{t-1}$.

When $(*)$ first occurs, Painter deletes $T \cap M$. Subsequently, Painter continues to use $\mathcal{S}$ (some vertices of $G$ may have an extra token). It suffices to show that (1) condition $\left(^{*}\right)$ must occur before Lister can win, and (2) after the round when $(*)$ occurs, each vertex of $T-M$ has more tokens remaining than the number of vertices remaining in $G$. By degeneracy, the rest of $T$ can be ignored, and continuing to use $\mathcal{S}$ enables Painter to win.

(1): We show that Lister cannot win without $\left(^{*}\right)$ occurring. That is, while $\left(^{*}\right)$ has not occurred, Lister cannot mark a vertex $v$ of $T$ more than $k$ times. When $v$ is marked and $(*)$ has not occurred, each round that marked $v$ has omitted at least one vertex of $T$ that was not yet omitted $\mu$ times. Hence $v$ has been marked fewer than $(t-1) \mu$ times. Since $(t-1) \mu \leq k$ and $v$ is any marked vertex of $T$, Lister has not won.

(2): Suppose that $(*)$ occurs when Lister plays $M$ in round $r$. A vertex $v \in T-M$ still has at least $k+1-(r-\mu)$ tokens. This value exceeds $\frac{t}{t-1} k-r$. Since $d_{G}(v)=|V(G)|$, and at least one vertex is deleted from $V(G)$ on each of these $r$ rounds, the remaining degree of $v$ is at most $\frac{t}{t-1} k-r$. 
Definition 2.3. Let $G$ be a complete multipartite graph with $t$ distinct sizes of parts, $k_{1}, \ldots, k_{t}$, where there are $r_{i}$ parts of size $k_{i}$. Following [7], we denote $G$ by $K_{k_{1} * r_{1}, \ldots, k_{t} * r_{t}}$. In addition, when $r_{i}=1$, we drop " $* r_{i}$ " from the notation.

Erdős, Rubin, and Taylor [2] proved that $K_{2 * r}$ is chromatic-choosable, and $K_{2 * r}$ was shown to be chromatic-paintable in [7]. Theorem 2.2 yields this immediately using $t=2$.

Corollary 2.4. If $G$ is chromatic-paintable, and $|V(G)| \leq 2 \chi(G)$, then $G \triangleleft \bar{K}_{2}$ is chromatic-paintable. In particular, $K_{2 * r}$ is chromatic-paintable.

Theorem 2.2 also yields chromatic-paintability for some other complete multipartite graphs, providing partial results toward Conjecture 1.2. Let $\mathcal{G}_{a}$ denote the class of graphs having an optimal coloring in which each color class has size at most $a$. Corollary 2.4 proves Conjecture 1.2 for graphs in $\mathcal{G}_{2}$. We next prove chromatic-paintability for a subset of $\mathcal{G}_{3}$. By Proposition 2.1(a), it suffices to consider complete multipartite graphs.

Corollary 2.5. $K_{1 * q, 2 * r, 3 * s}$ is chromatic-paintable for $q \geq 1$ and $3 s \leq q+3$.

Proof. If $K_{1 * q, 3 * s}$ is chromatic-paintable and $q+3 s \leq 2(q+s)$, then adding independent sets of size 2 preserves chromatic-paintability, as observed above. The inequality reduces to $s \leq q$, which holds when $3 s \leq q+3$ with $q \geq 1$ and $s$ is an integer. Therefore, it suffices to show that $K_{1 * q, 3 * s}$ is chromaticpaintable when $3 s \leq q+3$.

We start with $K_{1 * q}$ and iteratively take the join with independent sets of size 3. Consider $G \triangleleft \bar{K}_{3}$. To apply Theorem 2.2, we need $|V(G)| \leq$ $(3 / 2) \chi_{p}(G)$. By induction on $s, K_{1 * q, 3 * s}$ will be chromatic-paintable if $q+$ $3(s-1) \leq(3 / 2)(q+s-1)$, which simplifies to $3 s \leq q+3$.

Here there are at most $2 k+1-\frac{2}{3} q$ vertices, where $k$ is the chromatic number. We require $q \geq 1$ because $K_{2 * r, 3}$ is not 3-paintable for $r>1$ [7].

Ohba [11] proved chromatic-choosability when $|V(G)| \leq \chi(G)+\sqrt{2 \chi(G)}$. Using Theorem 2.2, we obtain chromatic-paintability in a larger class.

Theorem 2.6. If $|V(G)| \leq \chi(G)+2 \sqrt{\chi(G)-1}$, then $G$ is chromaticpaintable.

Proof. Let $n=|V(G)|$ and $k=\chi(G)$. A proper $k$-coloring of $G$ expresses $G$ as a subgraph of a complete $k$-partite graph, so we may assume that $G$ is a complete $k$-partite graph. Let $q$ be the number of parts of size 1 . We prove the claim by induction on $k-q$. When $k-q=1, G$ has the form $\bar{K}_{t} \oplus K_{q}$, which is chromatic-paintable by repeatedly applying Theorem 2.2 , adding one vertex each time. 
When $k-q>1$, let $T$ be a smallest non-singleton part, with $t=|T|$, and let $G^{\prime}=G-T$. It suffices to prove (1) $G^{\prime}$ is small enough for the induction hypothesis to make $G^{\prime}$ chromatic-paintable, and (2) $t$ is small enough for Theorem 2.2 to apply when $T$ is added to $G^{\prime}$.

(1): We are given $n \leq k+2 \sqrt{k-1}$ and need $n-t \leq(k-1)+2 \sqrt{k-2}$. Thus it suffices to prove $k+2 \sqrt{k-1}-t \leq k-1+2 \sqrt{k-2}$. This inequality simplifies to $t-1 \geq 2(\sqrt{k-1}-\sqrt{k-2})$. The difference of consecutive square roots is small; since $t \geq 2$ and $k \geq 3$, the inequality holds.

(2): Again we are given $n \leq k+2 \sqrt{k-1}$, but now we need $n-t \leq$ $\frac{t}{t-1}(k-1)$. It suffices to prove $k+2 \sqrt{k-1}-t \leq \frac{t}{t-1}(k-1)$, which simplifies to $2 \sqrt{k-1} \leq t-1+\frac{k-1}{t-1}$. The right side is minimized when $t-1=\sqrt{k-1}$, and there equality holds.

The bound in Theorem 2.6 holds with equality when $k-1$ is a perfect square and $G$ is the complete $k$-partite graph with $k-2$ parts of size 1 and two parts of size $1+\sqrt{k-1}$.

Consider now $G \oplus K_{t}$. Although always $\chi\left(G \oplus K_{t}\right)=\chi(G)+t$, adding $t$ vertices need not increase the paint number by $t$. In fact, $G \diamond K_{t}$ is chromaticpaintable when $t$ is sufficiently large. Kozik, Micek, and Zhu [9] also proved this, but without an explicit bound on the value of $t$ that suffices. We prove a technically more general statement; it applies to all graphs because always $G$ is $d$-degenerate when $d$ is the maximum degree.

Let $[k]$ denote $\{1, \ldots, k\}$. When $\chi(G)=k$, a proper $k$-coloring is an optimal coloring.

Theorem 2.7. Let $G$ be a d-degenerate graph having an optimal coloring with color classes $V_{1}, \ldots, V_{k}$ such that $\left|V_{i}\right| \leq a$ for $i \in[k]$. If $t \geq(a+1) d$, then $\chi_{p}\left(G \oplus K_{t}\right)=\chi\left(G \oplus K_{t}\right)$.

Proof. Let $\pi$ be an ordering of $V(G)$ in which each vertex has at most $d$ earlier neighbors. Let $T$ be the set of $t$ added dominating vertices not in $G$. Since $\chi\left(G \oplus K_{t}\right)=k+t$, it suffices to give a strategy for Painter to show that $G \rtimes K_{t}$ is $(k+t)$-paintable.

When $M \subseteq V(G)$, Painter deletes an independent subset of $M$ chosen greedily with respect to $\pi$. For $v \in V(G)$, in at most $d$ such rounds $v$ is marked but not removed, by the choice of $\pi$. Hence we will reserve $d+1$ tokens for such rounds; they are not used on rounds with $M \cap T \neq \varnothing$. When $M \cap T \neq \varnothing$, Painter will delete a vertex of $M \cap T$ or a subset of $M \cap V(G)$. We must ensure that the first option is not used too often when $v \in M$; the second option causes no trouble if $d+1$ tokens are reserved for $v$.

Let $v$ be a vertex of $G$ remaining when round $s$ begins. Let $g(v, s)$ denote the number of earlier steps on which $M \cap T \neq \varnothing$ and $v \notin M$. Let $V_{i, s}=\{v \in$ 
$\left.V_{i} \cap V\left(G_{s}\right): g(v, s) \leq d\right\}$. Intuitively, $V_{i, s}$ is the set of vertices in class $V_{i}$ that do not yet have $d+1$ tokens reserved for use within $G$. Always $V_{i, s+1} \subseteq V_{i, s}$.

When $M \cap T \neq \varnothing$, if $V_{i, s} \subseteq M$ for some $i$, then Painter deletes $V_{i, s}$ for some such $i$. Otherwise, Painter deletes a vertex of $M \cap T$.

Claim 1: While $\bigcup_{i} V_{i, s} \neq \varnothing$, at most ad $+k-1$ rounds with $M \cap T \neq \varnothing$ have been played. On at most $k-1$ such rounds, Painter deleted a nonempty set $V_{i, s}$, since a nonempty one remains. When Painter deletes a vertex of $M \cap T$, some vertex of $V_{i, s}$ is not in $M$. Since $\left|V_{i}\right| \leq a$, this happens at most ad times while $V_{i, s}$ remains nonempty.

Claim 2: While $v \in V_{i, s}$, there remain more than $d$ tokens at $v$. Since $t \geq(a+1) d$, vertex $v$ starts with at least $(a+1) d+k$ tokens. By Claim 1 , at most $a d+k-1$ rounds were played with $M \cap T \neq \varnothing$. Hence at least $d+1$ tokens remain available at $v$.

Claim 3: $A$ vertex of $T$ loses at most $k+t$ tokens before removal. A vertex of $T$ loses tokens only when $M \cap T \neq \varnothing$. On such rounds, Painter deletes a vertex of $T$ or the set $V_{i, s}$ for some $i$. There are at most $t$ rounds of the first type and at most $k$ of the second type.

By Claim 3, all of $T$ is removed. By Claim 2, $d+1$ tokens get reserved at each vertex of $G$. Hence the Painter strategy succeeds.

\section{Complete bipartite graphs}

Vizing [18] proved that $K_{k, r}$ is $k$-choosable if and only if $r<k^{k}$. We extend this characterization to $k$-paintability by considering a more general $f$-paintability problem on $K_{k, r}$. The theorem leads to further results about the $k$-paintability of $K_{k+j, r}$.

Theorem 3.1. Consider $K_{k, r}$ with $k \leq r$, having parts $X$ and $Y$ with $X=x_{1}, \ldots, x_{k}$. When $f\left(x_{i}\right)=t_{i}$ and $f(y)=k$ for $y \in Y$, Painter has a winning strategy if and only if $r<\prod t_{i}$.

Proof. (Necessity) It suffices to show that $K_{k, r}$ is not $f$-choosable when $r=\prod t_{i}$, Let $L\left(x_{i}\right)=U_{i}$ so that $\left|U_{i}\right|=t_{i}$ and $U_{1}, \ldots, U_{k}$ are pairwise disjoint. Let $\{L(y): y \in Y\}=U_{1} \times \cdots \times U_{k}$. Any coloring chosen from these lists puts a color from $U_{i}$ on $x_{i}$ for $1 \leq i \leq k$, but then the vertex in $Y$ having this set as its list cannot be properly colored.

(Sufficiency) Note that $r<\prod t_{i}$ requires $\min t_{i} \geq 1$. We use induction on $\sum t_{i}$. When $\sum t_{i}=k$, we have $Y=\varnothing$, and Painter will win. For $\sum t_{i}>k$, consider the first marked set $M$. By Proposition 2.1(c), we may assume that $M$ intersects both $X$ and $Y$. We may also assume $|M \cap X|=1$; otherwise, 
Painter deletes $M \cap X$ and each remaining vertex in $Y$ has more tokens than its degree. We may assume $M \cap X=\left\{x_{k}\right\}$. Let $q=|M \cap Y|$.

Case 1: $q<\prod_{i=1}^{k-1} t_{i}$. Painter deletes $v_{k}$; there remain $k-1$ vertices in $X$. By degeneracy, vertices of $Y-M$ are now irrelevant. Each of the $q$ vertices of $Y \cap M$ has $k-1$ tokens; the induction hypothesis applies.

Case 2: $q \geq \prod_{i=1}^{k-1} t_{i}$. Painter deletes $M \cap Y$. Since $r<\prod_{i=1}^{k} t_{i}$, the number of vertices left in $Y$ is less than $\prod_{i=1}^{k} t_{i}-\prod_{i=1}^{k-1} t_{i}$, which equals $\left(t_{k}-1\right) \prod_{i=1}^{k-1} t_{i}$. Since $t_{k}-1$ tokens remain on $v_{k}$, the induction hypothesis applies.

Corollary 3.2. $K_{k, r}$ is $k$-paintable if and only if $r<k^{k}$.

Hoffman and Johnson [4] proved that $K_{k+1, r}$ fails to be $k$-choosable if and only if $r \geq k^{k}-(k-1)^{k}$. We show in Corollary 3.8 that the least $r$ such that $K_{k+1, r}$ is not $k$-paintable is smaller than this when $k \geq 4$ (also for $k=3$, by computer search). Let $K_{l, r}$ have partite sets $X$ of size $l$ and $Y$ of size $r$. We present a recursive strategy for Lister on $K_{l, r}$ when each vertex in $Y$ has $k$ tokens and the vertices in $X$ have $t_{1}, \ldots, t_{l}$ tokens, respectively.

Definition 3.3. Fix nonnegative integers $k$ and $l$. Given an $l$-tuple $t$ of nonnegative integers, and $S \subseteq[l]$, let $t^{S}$ denote the $l$-tuple obtained from $t$ by reducing by 1 the coordinates indexed by $S$, and let $\left.t\right|_{\bar{S}}$ denote the $(l-|S|)$-tuple obtained from $t$ by restricting $t$ to the coordinates indexed by $[l]-S$. Define $g(k, t)$ recursively by letting

$$
g(k, t)= \begin{cases}0 & \text { if } \prod t_{i}=0, \\ 1 & \text { if } k=0, \\ \infty & \text { if } l<k, \\ \min _{S \subseteq[l]}\left[g\left(k-1,\left.t\right|_{\bar{S}}\right)+g\left(k, t^{S}\right)\right] & \text { otherwise }\end{cases}
$$

Proposition 3.4. If $l=k$, then $g(k, t)=\prod_{i=1}^{l} t_{i}$.

Proof. We use induction on $\prod t_{i}$. By definition, the claim holds when min $t_{i}=$ 0 . Otherwise, the boundary cases imply that in the nontrivial case the minimum occurs only when $|S|=1$. By symmetry, we may assume $S=\{l\}$. By the induction hypothesis, $g(k, t)=\prod_{i=1}^{l-1} t_{i}+\left(t_{l}-1\right) \prod_{i=1}^{l-1} t_{i}=\prod_{i=1}^{l} t_{i}$.

Thus the next theorem includes, within the special case $l=k$, a proof that $K_{k, r}$ is not $k$-paintable when $r \geq k^{k}$.

Theorem 3.5. Consider $K_{l, r}$ with $l \leq r$, having parts $X$ and $Y$ with $X=$ $x_{1}, \ldots, x_{l}$. When $f\left(x_{i}\right)=t_{i}$ and $f(y)=k$ for $y \in Y$, Lister has a winning strategy if $r \geq g(k, t)$. 
Proof. We give a recursive strategy for Lister, using induction on $\prod t_{i}$. If $\min t_{i}=0$, then Lister wins by playing a vertex in $X$ with no tokens, even if $Y$ is empty.

When $\min t_{i}>0$, we may assume $r=g(k, t)$ and let $S$ be an index subset of $[l]$ that yields the minimum in the definition of $g(k, t)$. Lister plays $\left\{x_{i}: i \in S\right\}$ plus $g\left(k-1,\left.t\right|_{\bar{S}}\right)$ vertices in $Y$. By Proposition 2.1(a), Painter deletes a maximal independent subset of $M$.

Case 1: Painter deletes $M \cap X$. Lister continues play on $(X-M) \cup$ $(M \cap Y)$, ignoring the vertices of $Y-M$. Each vertex of $M \cap Y$ now has $k-1$ tokens. Since $|M \cap Y|=g\left(k-1,\left.t\right|_{\bar{S}}\right)$, the induction hypothesis implies that Lister has a winning strategy in the remaining game.

Case 2: Painter deletes $M \cap Y$. Lister continues play on the remaining graph, with vertices $(Y-M) \cup X$. The token-count vector on $X$ is now $t^{S}$. Since $Y-M=g\left(k, t^{S}\right)$, the induction hypothesis implies that Lister has a winning strategy in the remaining game.

When Painter deletes $M \cap Y$, Lister would prefer to have marked as many vertices of $X$ as possible to obtain the maximum reduction in tokens on $X$. The danger, of course, is that when $M \cap X$ is larger, Painter may then decide to delete $M \cap X$.

Theorem 3.5 provides only an upper bound on the least $r$ such that Lister has a winning strategy. Ignoring $Y-M$ when Painter deletes $M \cap X$ limits Lister's options; possibly Lister should use these vertices. By Proposition 2.1(b), we may assume that $|S| \leq j+1$. This restriction on Lister allows us to compute upper bounds for larger examples.

Example 3.6. One approach for Lister is to always mark $l-k+1$ vertices in $X$ with the most tokens. This strategy is optimal when $l=k$ (always $|M \cap X|=1$, but it did not matter in Proposition 3.4 which was marked).

Consider $K_{k+1, r}$, where $l=k+1$ and each $t_{i}$ is $k$. If $|M \cap X|=2$ and Painter deletes $M \cap X$, then the remaining game has the form in Theorem 3.1. Lister can win it if $\prod_{i \notin S} t_{i}$ vertices remain (with $k-1$ tokens each) in $M \cap Y$ (that is, $|M \cap Y|=g\left(k-1,\left.t\right|_{\bar{S}}\right)$ ). By making $M \cap Y$ this size, Lister forces Painter to delete $M \cap Y$ to avoid losing.

By summing instances of $\prod_{i \notin S} t_{i}$ as the token counts in $X$ decrease, we can accumulate enough vertices in $Y$ to ensure a win for Lister. Eventually some token count in $X$ is driven to 0 , and Lister wins by marking that vertex with no need for additional vertices in $Y$. We thus obtain a value of $r$ such that $K_{k+1, r}$ is not $k$-paintable. Below we list the computation for $k \leq 4$. At each step, we list the vector $t$ of token counts in $X$ and the number of vertices in $Y$ that Lister will mark and Painter will delete. 


\begin{tabular}{llllll}
$k=2$ & & $k=3$ & & $k=4$ & \\
\hline$(2,2,2)$ & 2 & $(3,3,3,3)$ & 9 & $(4,4,4,4,4)$ & 64 \\
$(1,1,2)$ & 1 & $(2,2,3,3)$ & 4 & $(3,3,4,4,4)$ & 36 \\
& & $(2,2,2,2)$ & 4 & $(3,3,3,3,4)$ & 27 \\
& & $(1,1,2,2)$ & 1 & $(2,3,3,3,3)$ & 18 \\
& & $(1,1,1,1)$ & 1 & $(2,2,2,3,3)$ & 8 \\
& & & & $(2,2,2,2,2)$ & 8 \\
& & & & $(1,1,2,2,2)$ & 2 \\
& & & & $(1,1,1,1,2)$ & 1 \\
Total & 3 & Total & 19 & Total & 164
\end{tabular}

We conclude that $K_{3,3}$ is not 2-paintable, $K_{4,19}$ is not 3-paintable, and $K_{5,164}$ is not 4-paintable. In general, the threshold on $r$ for $\chi_{p}\left(K_{k+1, r}>k\right.$ is smaller than the threshold $k^{k}-(k-1)^{k}$ for $\chi_{\ell}\left(K_{k+1, r}>k\right.$ [4] (for $\chi_{\ell}\left(K_{4, r}\right)>$ 3 it is $r=19$, but for $\chi_{\ell}\left(K_{5, r}\right)>4$ it is $r=175$, larger than 164).

In fact, Lister can win even with smaller $r$. Exhaustive computer search of the games has shown that $K_{4,12}$ is not 3-paintable, improving the bound $r \leq 19$ computed above $\left(K_{4,11}\right.$ is 3 -paintable). We have not determined the least $r$ such that $K_{5, r}$ is not 4-paintable. However, optimizing over $S$ to compute the recursive bound $g(4,(4,4,4,4,4))$ from Theorem 3.5 shows that $K_{5,126}$ is not 4-paintable, improving on $K_{5,164}$.

Marking the vertices in $X$ with highest token counts minimizes the product of the remaining counts. This is a good heuristic, but sometimes Lister does better by marking vertices with smaller counts. We also found instances where Lister should mark fewer than $l-k+1$ vertices in $X$. These anomalies suggest that computing $\chi_{p}\left(K_{l, r}\right)$ is very hard; we only present bounds.

Proposition 3.7. If $t$ is the $(k+1)$-tuple $(k, \ldots, k)$, then $h(t) \leq \frac{k+1}{2 k}\left(k^{k}-\right.$ $1)+k^{k-1}$, where $h$ is the result of the recursive computation in Definition 3.3 when $S$ always corresponds to two vertices in $X$ with the most tokens.

Proof. The iteration to compute $h$ takes no more than $\frac{(k-1)(k+1)}{2}+1$ rounds. Each round accumulates the product of all remaining entries except the two largest, and those largest decrease by 1 . To get an upper bound on the product at each round, replace all entries with their average. Summing over $0 \leq n \leq(k-1)(k+1) / 2$, we have

$$
\begin{aligned}
h(t) \leq \sum\left(k-\frac{2 n}{k+1}\right)^{k-1} & \leq k^{k-1}+\int_{0}^{\frac{(k-1)(k+1)}{2}}\left(k-\frac{2 x}{k+1}\right)^{k-1} d x \\
& =\frac{k+1}{2 k}\left(k^{k}-1\right)+k^{k-1} .
\end{aligned}
$$


Corollary 3.8. When $k$ is sufficiently large, $K_{k+1, r}$ is $k$-choosable when $r<(.62+o(1)) k^{k}$, but $K_{k+1, r}$ is not k-paintable when $r>(.5+o(1)) k^{k}$.

Proof. The threshold $k^{k}-(k-1)^{k}$ for non- $k$-choosability [4] is asymptotic to $k^{k}\left(1-e^{-1}\right)$. On the other hand, the bound from Proposition 3.7 is less than $\frac{1}{2} k^{k}(1+3 / k)$.

\section{Paint-critical graphs}

Voigt in [19] characterized 3-choice-critical graphs, using the characterization of 2-choosable graphs. Using an analogous characterization of 2paintable graphs [20], we adapt the methods of Voigt to characterize 3paint-critical graphs.

Definition 4.1. A graph $G$ is $k$-paint-critical if $\chi_{p}(G)>\chi_{p}(G-e)$ whenever $e \in E(G)$. The core of a graph is the subgraph obtained by iteratively deleting vertices of degree 1.

Theorem 4.2 (Zhu [20]). A graph is 2-paintable if and only if each component of its core is $K_{1}$, an even cycle, or $\Theta_{2,2,2}$.

The difference between 2-choosability and 2-paintability is that a graph whose core is $\Theta_{2,2,2 k}$ with $k>1$ is 2-choosable but not 2-paintable.

Theorem 4.3. A graph is 3-paint-critical if and only if it is one of the following

1. An odd cycle.

2. Two edge-disjoint even cycles connected by a path.

3. $\Theta_{2 r, 2 s, 2 t}$ with $r>1, s \geq 1, t \geq 1$.

4. $\Theta_{2 r+1,2 s+1,2 t+1}$ with $r \geq 1, s \geq 1, t \geq 0$.

5. $\Theta_{2,2,2,2}$.

Proof. It suffices to consider connected graphs. Let $\mathcal{P}$ be the family of graphs listed above. Graphs in $\mathcal{P}$ are not 2-paintable, since they are cores but not in the list of Theorem 4.2. They are 2-degenerate, however, so they are 3paintable. To show that they are 3-paint-critical, it suffices to check that deleting any edge from any graph in $\mathcal{P}$ yields a graph whose core is named in Theorem 4.2. From $C_{2 k+1}$, we get $K_{1}$. From two even cycles joined by a path, we get one even cycle or disjoint even cycles. From a theta-graph consisting of three paths whose lengths have the same parity, we get an even cycle. From $\Theta_{2,2,2,2}$, we get $\Theta_{2,2,2}$.

It remains to show that every 3-paint-critical graph $G$ is in $\mathcal{P}$. Note first that $G$ is connected. Also $G$ has no vertex $v$ of degree 1; deleting $v$ would 


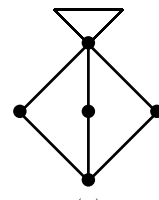

(a)

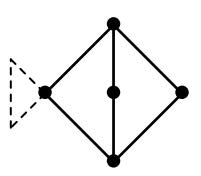

(b)

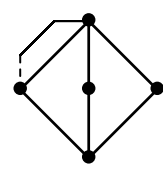

(c)

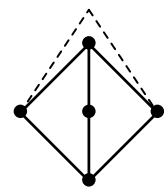

(d)

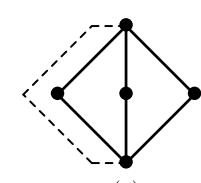

(e)

Figure 1: Ways to add an ear or closed ear to $\Theta_{2,2,2}$.

not reduce the paintability. Since $G-e$ is 2-paintable whenever $e \in E(G)$, each component of its core is $K_{1}$, an even cycle, or $\Theta_{2,2,2}$ (by Theorem 4.2). Since $G$ is connected, the core of $G-e$ has at most two components.

Suppose first that the core of $G-e$ has two components. If either is $K_{1}$, then $G$ has a vertex of degree 1, already excluded. Otherwise, each component has a cycle, and they are connected in $G$ by a path through $e$. Now $G$ has a proper non-2-paintable subgraph (and hence is not 3-critical) unless $G$ itself consists of two even cycles connected by a path.

We may therefore assume that the core of $G-e$ is connected. Hence $e$ is not a cut-edge of $G$, and $G$ has a cycle $C$ through $e$. If $G$ has no other cycle, then $G$ is a unicyclic graph with minimum degree at least 2 and hence is a cycle (in fact an odd cycle if not 2-paintable).

Hence $G$ has a cycle other than $C$. If $G$ has a cycle that shares no edges with $C$, then since $G$ is connected there is a path connecting them. As argued earlier, $G$ now properly contains a graph in $\mathcal{P}$ or belongs to $\mathcal{P}$.

Finally, suppose that $G$ has a cycle sharing an edge with $C$. Now $G$ contains a theta-graph consisting of three paths joining two vertices. If any two have lengths of opposite parity, then $G$ properly contains an odd cycle. Otherwise, the three lengths have the same parity. Now $G$ properly contains a theta-graph in $\mathcal{P}$ unless all three paths have length 2 . If $G=\Theta_{2,2,2}$, then $G$ is 2-paintable. Hence $\Theta_{2,2,2}$ occurs as a proper subgraph $G^{\prime}$ of $G$.

Since $G$ is 2-edge-connected, we can grow $G$ from any 2-edge-connected subgraph by iteratively adding ears (paths through new vertices that connect existing vertices) or closed ears (cycles using exactly one existing vertex). Consider growing $G$ from $G^{\prime}$ in this way. The possibilities for the first such addition are shown in Figure 1.

If a cycle is added or an added path forms a cycle with one edge of $G^{\prime}$ (cases (a), (b), and (c) in Figure 1), then the cycle has odd length or yields edge-disjoint even cycles.

If the added path connects the two high-degree vertices of $G^{\prime}$ (case (e)), then it forms an odd cycle or $\Theta_{2,2,2,2}$ (which lies in $\mathcal{P}$ ) or a graph containing $\Theta_{2 r, 2,2}$ with $r>1$ (again in $\mathcal{P}$ ). 
Finally, if the added path connects two low-degree vertices of $G^{\prime}$, then it forms an odd cycle with two edges of $G^{\prime}$ or forms a theta-graph of the form $\Theta_{2 r+1,3,1}$ with $r \geq 1$ having a high-degree vertex at an endpoint of the added path.

\section{References}

[1] N. Alon and M. Tarsi. Colorings and orientations of graphs. Combinatorica 12 (1992), 125-134. MR1179249

[2] P. Erdős, A. L. Rubin, and H. Taylor. Choosability in graphs. In: Proc. West Coast Conf. Combinatorics, Graph Theory and Computing (Humboldt State Univ., Arcata, Calif., 1979), Congr. Numer. 26 (1980), pp. 125-157. MR0593902

[3] J. Hladky, D. Kral, and U. Schauz. Brooks' Theorem via the Alon-Tarsi Theorem. Discr. Math. 310 (2010), 3426-3428. MR2721105

[4] D. G. Hoffman and P. D. Johnson Jr. On the choice number of $K_{m, n}$. In: Proc. 24th SE Intl. Conf. Comb., Graph Theory, and Computing (Boca Raton, FL, 1993), Congr. Numer. 98 (1993), pp. 105-111. MR1267344

[5] P.-Y. Huang, T.-L. Wong, and X. Zhu. Application of polynomial method to on-line list colouring of graphs. Europ. J. Combin. 33 (2011), 872-883. MR2889521

[6] H. A. Kierstead. On the choosability of complete multipartite graphs with part size three. Discrete Math. 211 (2000), 255-259. MR1735336

[7] S.-J. Kim, Y. Kwon, D. D. Liu, and X. Zhu. On-line list colouring of complete multipartite graphs. Electron. J.Combin. 19 (2012), Paper \#P41, 13 pages. MR2900416

[8] A. Kostochka, M. Stiebitz, and D. Woodall. Ohba's conjecture for graphs with independence number five. Discrete Math. 311 (2001), 9961005. MR2787311

[9] J. Kozik, P. Micek, and X. Zhu. Towards an on-line version Ohba's conjecture. Europ. J. Combin. 36 (2014), 110-121. MR3131879

[10] J. Noel, B. Reed, and H. Wu, A proof of a conjecture of Ohba. Preprint at arXiv:1211.1999 (8 November 2012).

[11] K. Ohba. On chromatic-choosable graphs. J. Graph Theory 40 (2002), 130-135. MR1899118 
[12] K. Ohba. Choice number of complete multipartite graphs with part size at most three. Ars Combinatoria 72 (2004), 133-139. MR2069052

[13] A. Riasat and U. Schauz. Critically paintable, choosable, or colorable graphs. Discrete Math. 312 (2012), 3373-3383. MR2961227

[14] B. Reed and B. Sudakov. List colouring when the chromatic number is close to the order of the graph. Combinatorica 25 (2004), 117-123. MR2109199

[15] J. Radhakrishnan and A. Srinivasan. Improved bounds and algorithms for hypergraph 2-coloring. Random Structures Algorithms 16 (2000), 4-32. MR1728350

[16] U. Schauz. Mr. Paint and Mrs. Correct. Electron. J. Combin. 16 (2009) \#R77. MR2515754

[17] U. Schauz. Flexible color lists in Alon and Tarsi's theorem, and time scheduling with unreliable participants. Electron. J. Combin. 17 (2010) \#R13. MR2578908

[18] V. G. Vizing. Vertex colorings with given colors (Russian). Diskret. Analiz. 29 (1976), 3-10.

[19] M. Voigt. On list Colourings and Choosability of Graphs. Habilitationsschrift, TU Ilmenau (1998).

[20] X. Zhu. On-Line List Colouring of Graphs. Electron. J. Combin. 16(1) (2009) \#R127. MR2558264

JAMES CARRAHER

Department of Mathematics

UNIVERSITY OF NEBRASKA-LINCOLN

LiNCOLN, NE

USA

SARAH LoEB

Department of Mathematics

UNIVERSITY OF ILLINOIS

URBANA, IL

USA

Thomas Mahoney

Department of Mathematics

UNIVERSITY OF ILLINOIS

URBANA, IL

USA 
Gregory J. Puleo

Department of Mathematics

UNIVERSITY OF ILLINOIS

URBANA, IL

USA

Mu-Tsun Tsai

Department of Mathematics

UNIVERSITY OF ILLINOIS

URBANA, IL

USA

Douglas B. West

Department of Mathematics

ZheJiang Normal University

JiNHUA, ZHEJIANG

China

AND

Department of Mathematics

UNIVERSITY OF ILLINOIS

URBANA, IL

USA

E-mail address: west@math.uiuc.edu

Received January 26, 2013 